\title{
Commentary \\ Spotlight on Accessory Proteins: RTK-RAS-MAPK Modulators as New Therapeutic Targets
}

\author{
Silke Pudewell and Mohammad Reza Ahmadian*
}

Citation: Pudewell, S.; Ahmadian, M.R. Spotlight on Accessory Proteins: RTK-RAS-MAPK Modulators as New Therapeutic Targets. Biomolecules 2021, 11, 895. https://doi.org/ 10.3390/biom11060895

Academic Editor: Daniel Abankwa

Received: 10 May 2021

Accepted: 15 June 2021

Published: 16 June 2021

Publisher's Note: MDPI stays neutral with regard to jurisdictional claims in published maps and institutional affiliations.

Copyright: (c) 2021 by the authors. Licensee MDPI, Basel, Switzerland. This article is an open access article distributed under the terms and conditions of the Creative Commons Attribution (CC BY) license (https:/ / creativecommons.org/licenses/by/ $4.0 /)$.
Institute of Biochemistry and Molecular Biology II, Medical Faculty, Heinrich-Heine University, 40225 Düsseldorf, Germany; sipud101@uni-duesseldorf.de

* Correspondence: reza.ahmadian@hhu.de

Abstract: The RTK-RAS-MAPK axis is one of the most extensively studied signaling cascades and is related to the development of both cancers and RASopathies. In the last 30 years, many ideas and approaches have emerged for directly targeting constituent members of this cascade, predominantly in the context of cancer treatment. These approaches are still insufficient due to undesirable drug toxicity, resistance, and low efficacy. Significant advances have been made in understanding the spatiotemporal features of the constituent members of the RTK-RAS-MAPK axis, which are linked and modulated by many accessory proteins. Given that the majority of such modulators are now emerging as attractive therapeutic targets, a very small number of accessory inhibitors have yet to be discovered.

Keywords: adaptor proteins; anchoring proteins; docking proteins; KRAS; scaffold proteins; RAF kinase; RTK; MEK; ERK

External signals are sensed, integrated, and amplified by conserved signaling cassettes. The activation of the RTK-RAS-MAPK signaling cascade is regulated by several different extracellular signals and intracellular proteins. Growth factors activate receptor tyrosine kinases (RTKs) at the plasma membrane, which in turn activate RAS via the GDP/GTP exchange reaction. These reactions are catalyzed by guanosine exchange factors (GEFs), such as SOS1 (son of sevenless 1). GTP-bound RAS initiates RAF dimerization and induces the phosphorylation cascade towards MEK and ERK [1]. Aberrant regulation or hyperactivation of the pathway leads to cancer and a group of developmental disorders with a mild gain-of-function of the RAS-MAPK pathway, which are collectively called RASopathies [2,3].

Targeting the constituent components of the RTK-RAS-MAPK signaling cascade often leads to high toxicity and activation of backup mechanisms, lowering the treatment efficacy and increasing the burden of the therapy. In this context, we have to consider that RASopathies are caused by germline mutations, therefore, most patients are children.

Here, we highlight a group of proteins named "accessory proteins", which are emerging as new potential therapeutic targets for the treatment of RAS-MAPK-related diseases. These proteins orchestrate the assembly and spatiotemporal localization of the constituent members of the cascade, without being part of the signaling pathway themselves [4]. Accessory proteins can be categorized into four distinct subgroups (see Figure 1): (1) anchoring proteins that bind to the membrane and other effectors (mostly kinases); (2) docking proteins that bind to receptors (e.g., RTKs and GPCRs) and more than one effector; (3) adaptor proteins that simply link two signaling components (e.g., receptor and GEF); and (4) scaffold proteins that bind two or more partners and provide a signaling platform. 


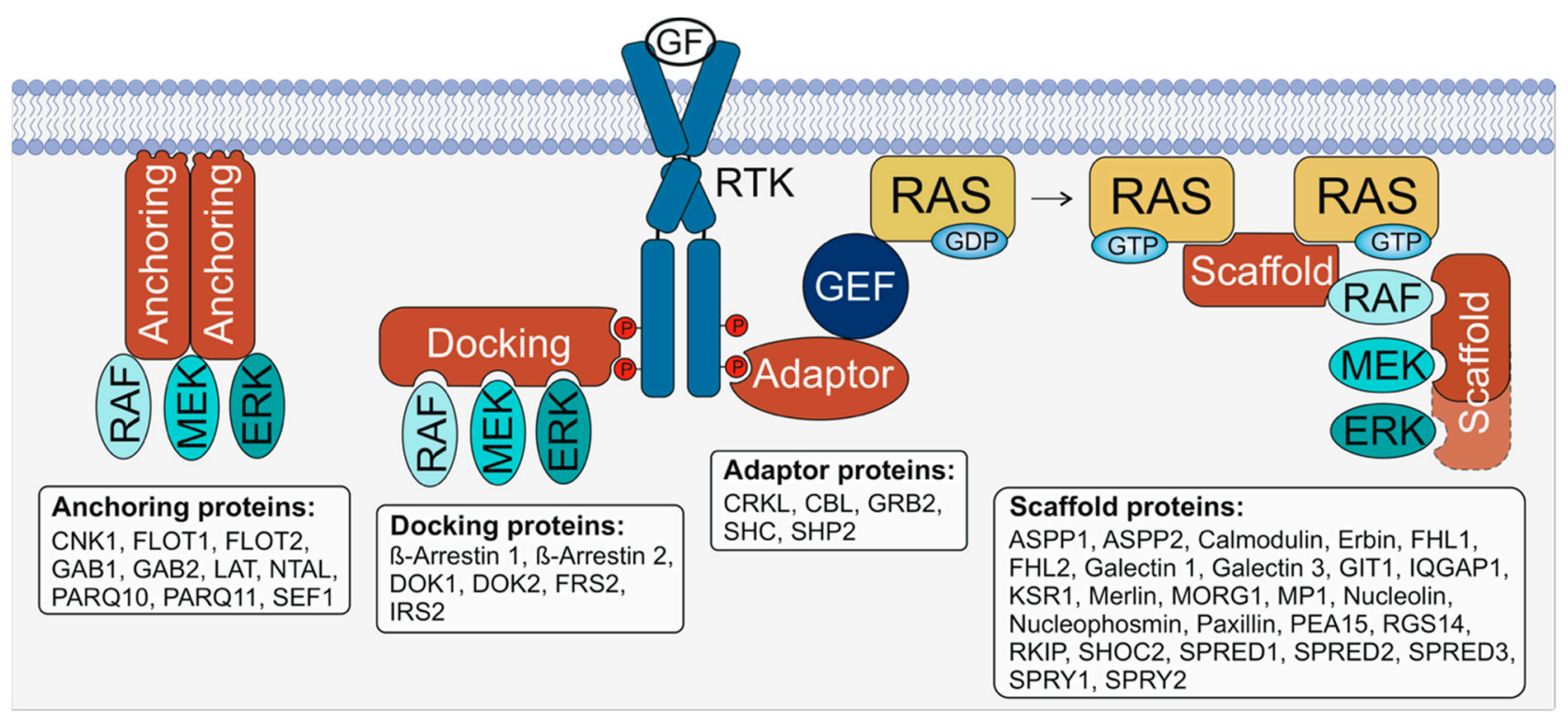

Figure 1. Accessory proteins can be divided into at least four subgroups: anchoring, docking, adaptor, and scaffold proteins. All groups include several different binders of the RTK-RAS-MAPK signaling pathway, such as RAF, MEK, and/or ERK, and often share common domains. Anchoring proteins include membrane-associated domains, such as pleckstrin homology (PH) and transmembrane (TM) domains or a posttranslational modification, (e.g., myristoylation in FLOT2) to determine a special subcellular localization and increase the dwell time of the proteins at the membrane. Docking proteins connect receptors with downstream effectors and feature receptor binding domains, such as PTBs (phosphotyrosine binding domains). FRS2, for example, interacts with the FGFR (fibroblast growth factor receptor) and links activated RTKs (receptor tyrosine kinases) with adaptor proteins such as GRB2 and SHC, and with other effectors, such as SOS1. Adaptor proteins can simply connect two proteins and often exhibit SH (SRC homology) 2 and SH3 domains. GRB2 is a well-known adaptor protein of RTKs and SOS1 but can also, as mentioned before, bind to other accessory proteins and RTKs and fine-tune the signaling machinery and the cross-talk of different pathways. Scaffold proteins can simultaneously bind several signaling components. They can contribute to clustering events by oligomerization via special domains or intrinsically disordered regions (such as galectin 1 and 3), recruit proteins to the site of action (such as SPRED1), or determine RAS signaling at a specific subcellular localization (such as MP1, which binds MEK1 and ERK1 on late endosomes). Scaffold proteins are also able to bind other accessory proteins and allow tight control of the RAS-MAPK pathway.

As modulators of the RTK-RAS-MAPK axis, accessory proteins are multidomain proteins that bind several interaction partners and connect them as a functional unit. Accordingly, they contribute to liquid-liquid phase separation (LLPS) events, which are crucial for a directed assembly of the respective signaling machinery [5,6]. Furthermore, they can fine-tune the crosstalk between signaling pathways, increase the dwell time of proteins on the membrane, induce nanoclustering, sequester effectors, and shield them from activation, or determine the cell type specificity and subcellular localization of signaling cassettes [7]. These modulating abilities turn accessory proteins into incredibly flexible and important proteins within a very specific context. In fact, it is easy to understand why the dysregulation of accessory proteins not only leads to cancer development and cancer progression in RAS-mutant tumors but also contributes to RASopathies.

The last 30 years of research have led to significant discoveries and improvements in cancer treatment, but new therapeutic approaches are still needed, especially for cancers with KRAS mutations. Several accessory proteins have been suggested to be promising targets in RAS-mutant cancer treatment, but a very limited number of inhibitors have yet to be discovered. The major advantage of targeting modulators rather than main players is that the hyperactive signaling mechanism is attenuated and reduced to a physiological level, but not abolished, through robust inhibition. For example, the knockout of KSR in mice does not abolish ERK phosphorylation completely, furthermore, it is quite well tolerated while mouse development and is resistant against RAS-driven tumor formation [8]. This 
can lead to less toxicity, particularly regarding side effects (including feedback mechanisms) and decrease the burden of the treatment [9]. An example of the advantage of targeting modulators is the case of the scaffold protein SHOC2; depletion of this protein leads to a better response with MEK inhibitor treatment by interfering with the feedback mechanism towards RAF [10]. SHOC2 binds PP1 and MRAS in a holoenzyme complex, which enables RAF dimerization by dephosphorylation and the release of RAF from its autoinhibited state. The knockout of SHOC2 in mice is embryonic lethal but quite well tolerated in adult animals and human cell lines, and the knockout of SHOC2 leads to growth inhibition of RAS-mutant cell lines [10]. In addition to cancer involvement of SHOC2, mutations of this protein were also detected in Mazzanti syndrome (a RASopathy) and in prenatalonset hypertrophic cardiomyopathy [11,12]. The mutations cause continuous membrane localization or enhanced binding of MRAS and PPP1CB, respectively, and highlight the fine-tuned signaling modulation of the scaffold protein. Another example is the anchoring protein CNK1. It localizes at the membrane via a pleckstrin homology $(\mathrm{PH})$ domain and binds RAF and RAS with different $\mathrm{C}$ - and $\mathrm{N}$-terminal domains, facilitating RAF activation and MAPK signaling [13]. The PH-domain inhibitor PHT-7.3 effectively prevents the colocalization of CNK1 with membrane-localized RAS and inhibits cell growth of KRASmutant cancer cell lines but not KRAS wild-type cell lines [14].

These two examples highlight the importance of a tightly controlled spatiotemporal organization of signaling components through accessory proteins and are just small insights into the large group of these modulators [4]. More research can pave the way for new therapeutic strategies involving single and cotreatment approaches that directly target the specific scaffold, adaptor, docking, or anchoring function of accessory proteins.

Funding: This study was supported by the Research Committee of the Medical Faculty of the Heinrich Heine University Düsseldorf (Grant number: 2020-70/9772617), the German Research Foundation (Deutsche Forschungsgemeinschaft or DFG; Grant number: AH 92/8-1), the German Research Foundation (Deutsche Forschungsgemeinschaft or DFG) through the International Research Training Group "Intra- and interorgan communication of the cardiovascular system" (Grant number: IRTG 1902-p6), the European Network on Noonan Syndrome and Related Disorders (NSEuroNet; Grant number: 01GM1621B); the German Federal Ministry of Education and Research (BMBF)German Network of RASopathy Research (GeNeRARe; Grant numbers: 01GM1902C).

Acknowledgments: We are grateful to our colleagues Farhad Bazgir, Neda Sadat Kazemein Jasemi, Niloufar Mosaddeghzadeh, and Christoph Wittich for stimulating discussion.

Conflicts of Interest: The authors declare no competing interests.

\section{References}

1. McKay, M.M.; Morrison, D.K. Integrating signals from RTKs to ERK/MAPK. Oncogene 2007, 26, 3113-3121. [CrossRef] [PubMed]

2. Castel, P.; Rauen, K.A.; McCormick, F. The duality of human oncoproteins: Drivers of cancer and congenital disorders. Nat. Rev. Cancer 2020, 20, 383-397. [CrossRef] [PubMed]

3. Tartaglia, M.; Gelb, B.D. Disorders of dysregulated signal traffic through the RAS-MAPK pathway: Phenotypic spectrum and molecular mechanisms. Ann. N. Y. Acad. Sci. 2010, 1214, 99-121. [CrossRef] [PubMed]

4. Pudewell, S.; Wittich, C.; Kazemein Jasemi, N.S.; Bazgir, F.; Ahmadian, M.R. Accessory proteins of the RAS-MAPK pathway: Moving from the side line to the front line. Commun. Biol. 2021, 4, 696. [CrossRef] [PubMed]

5. Lyon, A.S.; Peeples, W.B.; Rosen, M.K. A framework for understanding the functions of biomolecular condensates across scales. Nat. Rev. Mol. Cell Biol. 2020. [CrossRef]

6. Banani, S.F.; Lee, H.O.; Hyman, A.A.; Rosen, M.K. Biomolecular condensates: Organizers of cellular biochemistry. Nat. Rev. Mol. Cell Biol. 2017, 18, 285-298. [CrossRef] [PubMed]

7. Kolch, W. Coordinating ERK/MAPK signalling through scaffolds and inhibitors. Nat. Rev. Mol. Cell Biol. 2005, 6, 827-837. [CrossRef] [PubMed]

8. Nguyen, A.; Burack, W.R.; Stock, J.L.; Kortum, R.; Chaika, O.V.; Afkarian, M.; Muller, W.J.; Murphy, K.M.; Morrison, D.K.; Lewis, R.E.; et al. Kinase Suppressor of Ras (KSR) Is a Scaffold Which Facilitates Mitogen-Activated Protein Kinase Activation In Vivo. Mol. Cell. Biol. 2002, 22, 3035-3045. [CrossRef] [PubMed]

9. Matallanas, D.; Crespo, P. New druggable targets in the Ras pathway? Curr. Opin. Mol. Ther. 2010, 674-683. 
10. Jones, G.G.; Del Río, I.B.; Sari, S.; Sekerim, A.; Young, L.C.; Hartig, N.; Zubiaur, I.A.; El-Bahrawy, M.A.; Hynds, R.E.; Lei, W.; et al. SHOC2 phosphatase-dependent RAF dimerization mediates resistance to MEK inhibition in RAS-mutant cancers. Nat. Commun. 2019, 10, 1-16. [CrossRef] [PubMed]

11. Motta, M.; Giancotti, A.; Mastromoro, G.; Chandramouli, B.; Pinna, V.; Pantaleoni, F.; Di Giosaffatte, N.; Petrini, S.; Mazza, T.; D'Ambrosio, V.; et al. Clinical and functional characterization of a novel RASopathy-causing SHOC2 mutation associated with prenatal-onset hypertrophic cardiomyopathy. Hum. Mutat. 2019, 40, 1046-1056. [CrossRef] [PubMed]

12. Cordeddu, V.; Di Schiavi, E.; Pennacchio, L.A.; Ma'ayan, A.; Sarkozy, A.; Fodale, V.; Cecchetti, S.; Cardinale, A.; Martin, J.; Schackwitz, W.; et al. Mutation of SHOC2 promotes aberrant protein N-myristoylation and causes Noonan-like syndrome with loose anagen hair. Nat. Genet. 2009, 41, 1022-1026. [CrossRef] [PubMed]

13. Therrien, M.; Wong, A.M.; Kwan, E.; Rubin, G.M. Functional analysis of CNK in RAS signaling. Proc. Natl. Acad. Sci. USA 1999, 96, 13259-13263. [CrossRef] [PubMed]

14. Indarte, M.; Puentes, R.; Maruggi, M.; Ihle, N.T.; Grandjean, G.; Scott, M.; Ahmed, Z.; Meuillet, E.J.; Zhang, S.; Lemos, R.; et al. An inhibitor of the pleckstrin homology domain of CNK1 selectively blocks the growth of mutant KRAS cells and tumors. Cancer Res. 2019, 79, 3100-3111. [CrossRef] [PubMed] 